\title{
Generalised Epidermolysis Bullosa with Severe Anaemia in an Adolescent: A Case Report
}

\section{Shivam Jannawar, Sabahat Ahmed, Rajesh K Kulkarni and Deepali Ambike}

Department of Paediatrics, Yashwantrao Chavan Memorial - Post Graduate Institute, Pune, India

\section{Correspondence:}

Rajesh K Kulkarni

Department of Paediatrics,

Yashwantrao Chavan Memorial - Post Graduate Institute,

Pune, India.

Email: docrajesh75@yahoo.com

DOI: $10.3126 /$ jnps.v41i2.35059

Submitted on: $2020-08-06$

Accepted on: 2021-02-26

Acknowledgements: None

Funding: Nil

Conflict of Interest: None declared

Permission from IRB: Yes

To cite this article: Jannawar S, Ambike D, Ahmed S, Kulkarni RK, Ambike D. Generalised Epidermolysis Bullosa with Severe Anaemia in an Adolescent: A Case Report. J Nepal Paediatr Soc. 2021;41(2): 290-3.

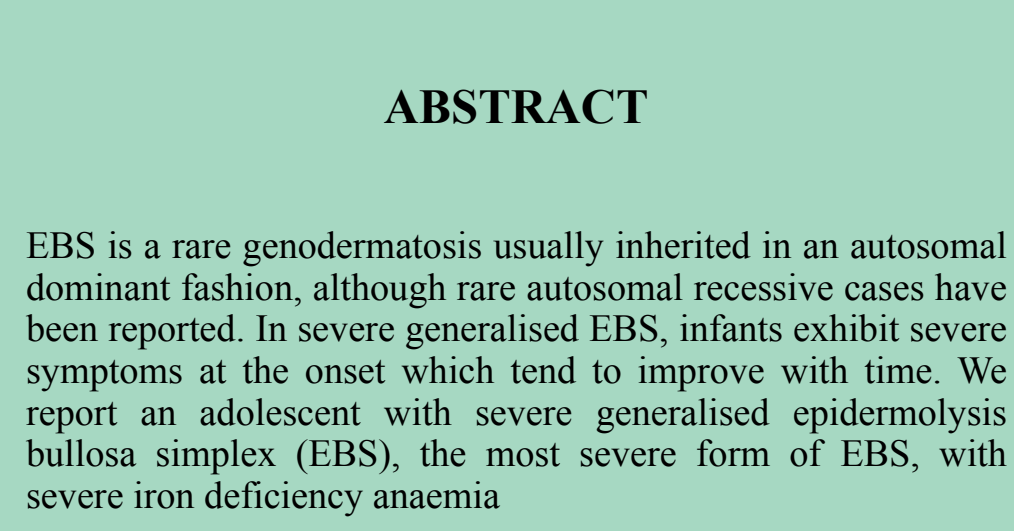

Keywords: Adolescent; Generalised Epidermolysis Bullosa; Severe anaemia 


\section{INTRODUCTION}

Weakness in children may have various etiologies Epidermolysis bullosa (EB) is a heterogeneous group of rare inherited connective tissue disorders characterised by marked fragility of epithelial tissues with prototypic blistering, erosions, and non-healing ulcers following minimal rubbing or frictional trauma. ${ }^{1} \mathrm{~EB}$ is classified into four major categories, each with many subtypes based on the precise location at which separation or blistering occurs, namely, epidermolysis bullosa simplex (EBS; intraepidermal skin separation), epidermolysis bullosa junctional [EBJ; skin separation in lamina lucida or central basement membrane zone (BMZ)], dystrophic epidermolysis bullosa or epidermolysis bullosa dystrophica (EBD; sublamina densa BMZ separation), and Kindler syndrome (multiple cleavage planes). ${ }^{2}$

The fundamental pathology of EB lies on the increase in collagenase activity, leading to collagen degeneration and hence splitting of various epidermal layers or at the transition between epidermis and dermis. ${ }^{3,4}$ EBS is the most common type of EB, accounting for 75 to $85 \%$ of cases of $\mathrm{EB}$ in the western world. ${ }^{4} \mathrm{EBS}$ is usually caused by pathogenic variants in the keratin genes (KRT5 and KRT14) with resultant formation of a cleavage plane at the level of the basal keratinocytes. ${ }^{5}$ Localised EBS (formerly known as WeberCockayne EBS), usually associated with little or no extracutaneous involvement, is the mildest and most common form of EBS. Nail dystrophy is rare and generally mild. Severe generalised EBS (formerly known as Dowling-Meara EBS) is the most severe form of EBS and presents with widespread friction-induced blistering at birth. Involvement of the oral mucosa and nail dystrophy are common. Generalised intermediate EBS (formerly known as Koebner EBS) may present at birth with blistering and possibly with milder clinical courses. ${ }^{6}$ Anaemia is common in children with EBS and is multifactorial.

In this report, we describe an adolescent with severe generalised EBS with severe iron deficiency anaemia.

\section{CASE REPORT}

A 12 - year - old girl presented with cough and generalised weakness for 10 days. She was apparently alright 10 days back when she started experiencing cough which was acute in onset, nonproductive in nature, not associated with breathlessness, fever and expectoration. She had generalised weakness since cough started. There was history of decreased oral acceptance.

Child had extensive skin lesions with blisters over the whole body noted soon after birth. Child had syndactyly of all fingers in all limbs. The skin was so delicate that it was prone for trauma even with the stretching of the clothes. Child was on liquid diet due to restriction in mouth opening as a result of extensive fibrosis. Child was diagnosed as generalised epidermolysis bullosa. Child had recurrent traumatic injurious lesions due to delicate skin. Parents used to do dressing of the lesion regularly. There was no history of similar skin disease in family. Child was born from uneventful normal vaginal delivery with no history of maternal or fetal complications. There was no history of any delayed milestones. Child goes to regular school with average scholastic performance.

On examination, she was conscious and oriented. There was severe pallor. Central pulses were bounding while the peripheral pulses were not palpable properly due to thickened and fibrosed skin. Scaling of skin was present all over the body. Syndactyly was present in both arms and legs. (Fig. $1,2)$.

A haemic murmur was appreciated with normal heart sounds on cardiac examination. There was no organomegaly. Laboratory reports on admission were suggestive of severe anaemia with haemoglobin of $1.7 \mathrm{gm} / \mathrm{dl}$ with severe microcytic and hypochromic red cell morphology. Iron studies showed low serum iron and ferritin $(15 \mathrm{mcg} / \mathrm{ml}$ and $10 \mathrm{ng} / \mathrm{ml}$ respectively). Vitamin $B_{12}$ range was within normal range. Chest radiograph showed cardiomegaly. All other investigations were found in normal range. Child was given packed RBC transfusion for the same. The cause of anaemia was thought to be due to repeated trauma to the skin and blood loss along with the poor appetite. Child was 


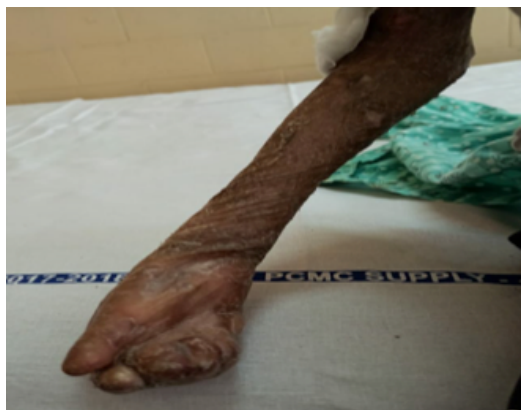

Figure 1. Thickened and fibrosed skin causing syndactyly in foot

discharged after nutritional counseling on oral iron supplements.

\section{DISCUSSION}

Severe generalised EBS is devastating both to patients and their families. Obstetricians and paediatricians must be familiar with the mode of inheritance, age-related morbidity and mortality associated with this rare but severe disease in order to provide timely counselling on the natural history of the disease, recurrence risk and reproductive options to the families. EBS is almost always inherited in an autosomal dominant fashion, although rare autosomal recessive forms have been reported. ${ }^{7}$ Knowledge of the exact genetics of EBS helps in counselling the families regarding the prognosis of their affected children and recurrence risk for future pregnancies.

Unlike EBD and EBJ, EBS is usually a milder disease and not associated with high mortality. ${ }^{8}$ Our patient had severe generalised EBS based on the onset of the disease at birth, disseminated friction or trauma-induced blistering, involvement of oral mucosa, and presence of nail dystrophy. Despite these findings, symptoms observed in severe generalised EBS tend to improve with time. ${ }^{8}$ The main causes of early morbidity and mortality in severe generalised EB are septicemia, malnutrition, and electrolyte disturbances. ${ }^{1-4}$ Hence, skin care and nutrition support must be meticulous. Malnutrition can be attributed to recurrent mucosal lesions, feeding difficulties, high energy

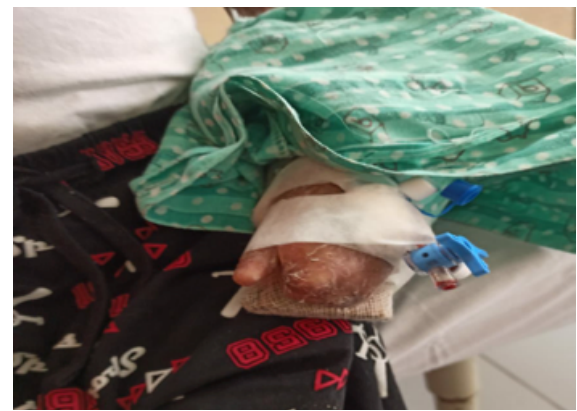

Figure 2. Thickened and fibrosed skin causing syndactyly in hand

consumption from accelerated skin turnover, transcutaneous loss of nutrients, and catabolic state from recurrent infections. ${ }^{8}$ It is therefore important to involve dietitians to prepare easy-to-consume recipes, identify high-caloric and protein-fortified foods and beverages to replace protein lost in draining blisters, suggest vitamin and mineral nutritional supplements and recommend dietary adjustments to prevent gastrointestinal problems, such as constipation, diarrhoea or painful defecation. ${ }^{4}$ During hospitalisation, the importance of adequate nutritional intake should be reinforced. When indicated, the option of gastrostomy should be discussed with the patient and his / her family for those patients who remain cachectic despite conservative measures.

\section{CONCLUSIONS}

Herein, we report child with severe generalised EBS. It is devastating for both parents and child to manage the disorder. Meticulous care of skin is necessary to prevent any chances of infections. Regular dressing of wounds is necessary to control blood loss and infections. Patient may require regular blood transfusion due to recurrent blood loss. Severe generalised EBS is an inherited blistering skin disease associated with significant morbidity and mortality.

\section{REFERENCES}

1. Chong S, Hon K, Yuen L, Choi P, Ng W, Chiu T. Neonatal epidermolysis bullosa: lessons to learn about genetic 
counseling. J Derma Treat. 2021;32:29-32. DOI: 10.1080/09546634.2018.1527999

2. Nakano A, Chao S, Pulkkinen L, Murrell D, Bruckner-Tuderman L, Pfendner E, et al. Laminin 5 mutations in junctional epidermolysis bullosa: molecular basis of Herlitz vs non-Herlitz phenotypes," Human Genetics, 2002;110:41-51. DOI: 10.1007/s00439-001-0630-1

3. Abu Sa'd J, Indelman M, Pfendner E, Falik-Zaccai TC, Mizrachi-Koren M, Shalev S, et al. Molecular epidemiology of hereditary epidermolysis bullosa in a Middle Eastern population. J Invest Dermatol. 2006;126(4):777-81. DOI: $10.1038 /$ sj.jid.5700163

4. Sheppard S, Anderson L, Sibbald L, Cotton C. Generalized, severe epidermolysis bullosa simplex caused by a Keratin 5 p.E477K mutation," Pediatr Derma. 2019:36(6);1007-1009. DOI: 10.1111/pde.13965.

5. Minakawa S, Nakano H, Nakajima K, Matsuzaki Y, Takiyoshi N, Akasaka E, et al. Mutational analysis on 16 Japanese population cases with epidermolysis bullosa simplex. J Dermatol Sci. 2013;72(3):330-2. DOI: 10.1016/ j.jdermsci.2013.08.001

6. Khani P, Ghazi F, Zekri A. Keratins and epidermolysis bullosa simple X. J Cell Physiol. 2018;234(1):289-97. DOI: $10.1002 /$ jcp. 26898

7. Kang T, Lee J, Kim S ,Oh S, Kim S. Novel and recurrent mutations in Keratin 5 and 14 in Koreanpatients with Epidermolysis bullosa simplex. J Dermatol Sci. 2010;57(2):90-4. DOI: 10.1016/j.jdermsci.2009.12.002

8. Allman S, Haynes L, MacKinnon P, Atherton D. Nutrition in dystrophic epidermolysis bullosa. Pediatr Dermatol. 1992;9(3):231-8. DOI: 10.1111/j.1525-1470.1992.tb00337.x 